\title{
Clinical Trial of Low Irritative Skin Care Cosmetics in Japanese Subjects with Dry Skin
}

This article was published in the following Dove Press journal:

Clinical, Cosmetic and Investigational Dermatology

\section{Kazunori Tanaka' \\ Teruaki Nagasawa' \\ Yuko Nomura ${ }^{2}$ \\ Yoshiki Kubota' \\ Akihiko Miyake' \\ Kimio Kawamura' \\ Yoko Yamaguchi'}

'Pharmaceutical Products Research and Development Section, NANOEGG® Research Laboratories, Inc, Kawasaki, Kanagawa, Japan; ${ }^{2}$ Nomura Dermatology Clinic, Yokohama, Kanagawa, Japan
Correspondence: Yoko Yamaguchi Pharmaceutical Products Research and Development Section, NANOEGG®

Research Laboratories, Inc., Innovation

Center of NanoMedicine 4F, 3-25-I4

Tono-Machi, Kawasaki-Ku, Kawasaki,

Kanagawa 210-082I, Japan

Tel +8I $44400 I I 55$

Fax +81366853127

Email rdm@nanoegg.co.jp
Purpose: To evaluate whether MediQOL, which was developed as a care cosmetic for sensitive skin, causes a change in skin condition for subjects with dry skin requiring moisture retention.

Subjects and Methods: This study includes 20 Japanese subjects with dry skin who required moisture retention, as identified by a dermatologist. The subjects used the novel MediQOL products twice a day for four consecutive weeks. A skin evaluation was performed by a dermatologist, and each subject completed a questionnaire prior to the study period and after two and four weeks of MediQOL use.

Results: After four weeks of MediQOL use, alleviation of skin dryness, redness, and itchiness was observed in the subjects. The water/oil content of the skin also improved during the study period.

Conclusion: Four weeks of MediQOL use resulted in the alleviation of skin dryness, redness, and itchiness and balanced the water/oil content of the skin. MediQOL is expected to be effective in improving the condition of various skin types, including oily, mixed, and sensitive skin as well as dry skin.

Keywords: skin care, dryness, erythema, humidity retention, quality of life

\section{Introduction}

In general, the skin barrier function is maintained by the horny layer, which consists of intercellular lipids, sebum, and corneocytes containing natural moisturizing factor. It is thought that deterioration of the skin barrier function leads to uncomfortable conditions of the skin. ${ }^{1-7}$ The causes of reduced skin barrier function include a disturbance of the turnover cycle that creates a new horny layer and a deficiency of the moisturizing ingredients in the horny layer. Several studies have reported a relationship between sensitive and dry skin and hypofunction of the skin barrier. $^{8-12}$

Supplementation of moisturizing ingredients such as ceramide, natural moisturizing factor, and oil is one method used to improve the skin barrier function. However, as the formation of the extracellular lipid lamellar membranes that contribute to the skin barrier function involves free fatty acids, ceramides, and cholesterol, ${ }^{13-16}$ a mere supplementation of specific ingredients via cosmetics is not optimum skin care. Rather, the replenishment of skin barrier components can lead to autonomous recovery of the skin barrier function. Thus, cosmetics containing essential components used to generate the horny layer, but not including ingredients not found on the skin such as surfactant, may be ideal products to improve the skin 
condition and quality of life (QOL) in subjects with hypofunction of the skin barrier.

We hypothesized that the direct supplementation of the components that support the adjustment of the horny layer turnover rate is important for subjects with various types of skin conditions such as dry, oily, and sensitive skin. We developed three novel cosmetics: MediQOL washing powder, consisting of analog components of sebum; MediQOL skin water, with a similar composition to the cell culture medium for keratinocytes; and MediQOL skin lipid, for the supplementation of sebum and intercellular lipids imitating the composition rate of healthy skin. To evaluate the impact of MediQOL on skin with reduced barrier function, we compared the skin conditions of subjects with dry skin who require skin moisturization before and after the use of MediQOL.

\section{Materials and Methods Subjects and Study Design}

This was an open trial to evaluate the safety and impact of novel skin care cosmetics on skin condition. The study protocol (Ex. 695) was approved by the Institutional Review Board for the Asai Dermatology Clinic and followed the principles of the Declaration of Helsinki and Ethical Guidelines for Medical and Health Research Involving Human Subjects (Public Notice of the Ministry of Education, Culture, Sports, Science and Technology and the Ministry of Health, Labor, and Welfare in Japan). This study was registered at UMIN (UMIN000035370). All subjects provided written informed consent. We enrolled 20 subjects who lived in Japan and in whom a dermatologist had identified the requirement for skin moisturization by visual examination.

The target areas of this study included the face and the body (the forearm or the lower leg). One side of the target areas on the forearm or the lower leg in each subject was designated as the test part, on which the MediQOL (NANOEGG ${ }^{\circledR}$ Research Laboratories, Inc., Kawasaki, Japan) was used, and the other side was designated as the control part, to which the subject's existing cosmetics routine was applied.

\section{Test Products and Usage}

MediQOL washing powder, MediQOL skin water 35/54, and MediQOL skin lipid 15/33II (NANOEGG® Research Laboratories, Inc., Kawasaki, Japan) were used in this study (Table 1). All subjects used the test products twice a day (morning and evening) for four consecutive weeks. Subjects washed their face with MediQOL washing powder, then conditioned with MediQOL skin water before moisturizing with MediQOL skin lipid. Only MediQOL skin water and MediQOL skin lipid were applied to the forearm or lower leg.

\section{Evaluation of Skin Condition}

Skin evaluations were performed by a dermatologist and questionnaires were completed by the subjects prior to the start of the study period (W0), after two weeks of MediQOL use (W2), and after four weeks of MediQOL use (W4). The skin conditions of erythema, papules, infiltrations, scars, lichenization, dryness, and pruritus were classified as severe, moderate, mild, minor, or none. The self-reported questionnaire included the following seven evaluation items: redness; itchiness; dryness; excess sebum; redness with physical stimulation such as rubbing the skin with clothing or a hand; worsening of the skin condition when tired and/or stressed; and burning, tingling, and/or redness after the use of MediQOL or other cosmetics routinely used by the subjects. These items were classified as occurring all of the time, some of the time, not very often, or never.

\section{Instrumental Analyses}

The measurements of transepidermal water loss (TEWL) and erythema were performed using a Cutometer MPA580 (Courage+Khazaka, Köln, Germany), and skin moisture and oil content were measured using a WSK-P500U (WaveCyber Corporation, Saitama, Japan). Measurements were obtained at four points within the target area that remained consistent between $\mathrm{W} 0, \mathrm{~W} 2$, and $\mathrm{W} 4$.

Photographs of the skin conditions on the body were recorded using a digital camera (WG-III Digital Camera, Pentax, Tokyo, Japan) and a digital microscope (DS-10D, Nidek Co., LTD., Aichi, Japan). A Robo Skin Analyzer (RSA 50SII, Shibuya Corporation, Ishikawa, Japan) was used for the analysis of the face.

All measurements and photographs were obtained after the target area was acclimatized for 15 minutes in a temperature- and humidity-controlled room, only the face was washed before acclimatization.

\section{Analysis Method}

The results of the skin evaluations and questionnaires were grouped as skin condition levels for each evaluation item at W0, W2, and W4. The average for each component of the 
Table I Composition of MediQOL Washing Powder, Skin Water, and Skin Lipid

\begin{tabular}{|l|l|l|}
\hline Type & Form & Composition \\
\hline $\begin{array}{l}\text { Washing } \\
\text { powder }\end{array}$ & Powder & $\begin{array}{l}\text { Potassium palmitate; Potassium stearate; } \\
\text { Potassium myristate; Potassium laurate }\end{array}$ \\
\hline $\begin{array}{l}\text { Skin } \\
\text { water 35/ }\end{array}$ & Liquid & $\begin{array}{l}\mathrm{H}_{2} \mathrm{O} \text {; L-Arginine monohydrochloride; } \\
\text { L-Histidine monohydrochloride; L-Isoleucine; } \\
\text { L-Leucine; L-Lysine monohydrochloride; } \\
\text { L-Methionine; L-Phenylalanine; L-Serine; } \\
\text { L-Threonine; L-Tryptophan; L-Tyrosine; } \\
\text { L-Valine; L-Alanine; L-Cysteine } \\
\text { monohydrochloride; L-Proline; Calcium } \\
\text { pantothenate; Folic acid; Inositol; } \\
\text { Niacinamide; Riboflavin; Pyridoxine } \\
\text { hydrochloride; Thiamine hydrochloride; } \\
\text { Biotin; Cyanocobalamine; Sodium pyruvate; } \\
\text { Thioctic acid; Thymidine; Potassium chloride; } \\
\text { Sodium chloride; Ferrous sulfate; Magnesium } \\
\text { chloride; Disodium phosphate; Zinc sulfate; } \\
\text { Glucose; Dipotassium glycyrrhizinate }\end{array}$ \\
\hline Skin lipid & Balm & $\begin{array}{l}\text { Tripalmitin; Hexyldecyl palmitate; Squalane; } \\
\text { Trimyristin; Triolein; Oleic acid oleyl; Isocetyl } \\
\text { myristate; Trilaurin; Glyceryl dioleate; } \\
\text { Methylheptyl laurate; Cholesterol; Ceramide } \\
\text { 6ll; Carnauba wax; Ceramide 2; Ceramide 3; } \\
\text { Behenic acid; Stearyl glycyrrhetinate }\end{array}$ \\
\hline
\end{tabular}

instrumental analyses at W0 was calculated and each measured value was classified as greater than the mean value or less than the mean value (moderate or mild groups, respectively). We compared the data obtained at W0, W2, and W4 for the face and body target areas separately.

Incomplete data due to adverse events or withdrawal of consent was included until the point of discontinuance.

\section{Statistical Analysis}

Data in this study were expressed as mean \pm standard error (SE). Statistical significance was evaluated using the paired Student's $t$-test for comparisons between the data of two time points. Statistical significance was set at $p<0.05$ and all analyses were performed using Excel (Microsoft, Redmond, Washington, U.S.).

\section{Results}

\section{Subject Characteristics}

There were 20 subjects enrolled in this study. Three subjects were men and 17 were women. The mean subject age was $44.4 \pm 10.9$ years (range: $21-63$ years). All subjects used MediQOL on the face, and four and 16 subjects also used MediQOL on the lower leg and forearm, respectively. No subjects chose to withdraw from the study; however, the dermatologist discontinued the facial use of MediQOL in three subjects after W2 due to its adverse effects.

\section{Skin Evaluation}

When compared to the condition of the facial skin at W0, less erythema, papules, infiltration, lichenization, dryness, scars, and pruritus were observed throughout the study period (Figure 1A).

As shown in Figure 1B, sequential relief of the symptoms of infiltration, scars, and dryness at the test part of the body was observed throughout the test period when compared to the skin conditions at W0. The degrees of erythema and pruritus in the test part of the body were decreased at W2 compared to W0, and this change was maintained at W4 (Figure 1B). Relief of the symptoms of papules and lichenization was observed at W4 (Figure 1B). These symptoms were more reduced in the test part of the subjects compared to the control part (Figure 1B).

The ratio of subjects classified as having a severe level of dryness changed from $5 \%$ and $15 \%$ at $\mathrm{W} 0$ in the face and on the test part of the body, respectively, to $0 \%$ at $\mathrm{W} 2$ in both (Figure 1). The ratio of subjects classified as having no dryness increased to $70 \%$ and $45 \%$ in the face and on the test part of the body after four weeks compared with $0 \%$ at $\mathrm{W} 0$ for both. The ratio of subjects classified as having no dryness at the control part was $25 \%$ at W4.

\section{Questionnaire results}

The ratio of subjects reporting itchiness, dryness, and excess sebum in the face gradually decreased throughout the study period (Figure 2A). Relief of redness was reported at W4 and relief of redness with physical stimulation was reported at W2 (Figure 2A). The ratio of subjects who reported redness, itchiness, and excess sebum all of the time decreased to $0 \%$ at $\mathrm{W} 4$ (Figure $2 \mathrm{~A}$ ). The ratio of subjects reporting no dryness in the face was $0 \%$ at W0 and $17.6 \%$ at W4 (Figure 2A).

The ratio of subjects reporting redness, itchiness, and dryness in the test part of the body decreased from W2 to W4, and the ratio of subjects who reported no dryness in the test part of the body increased from $0 \%$ at W0 to $15 \%$ at W4 (Figure 2B). There was little change in the ratio of subjects who reported excess sebum and redness with physical stimulation, and the degrees of the change in 
A
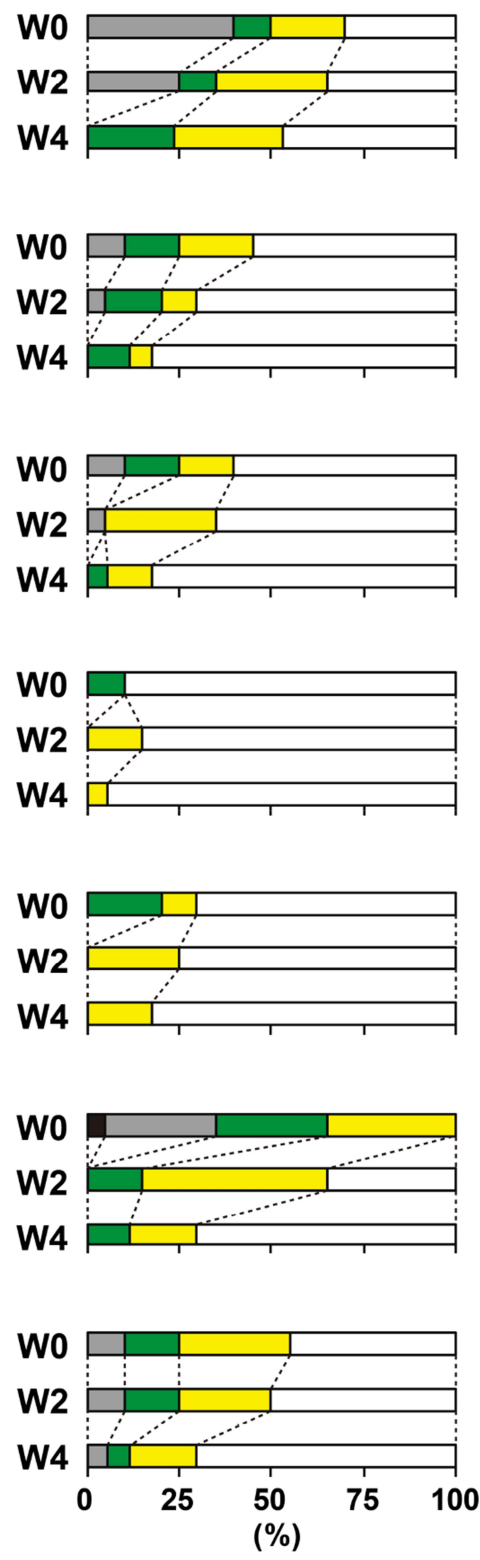

B

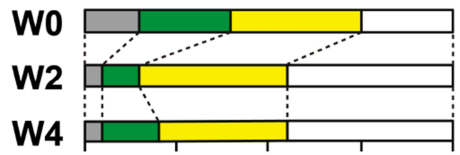

Wo

W2

W4
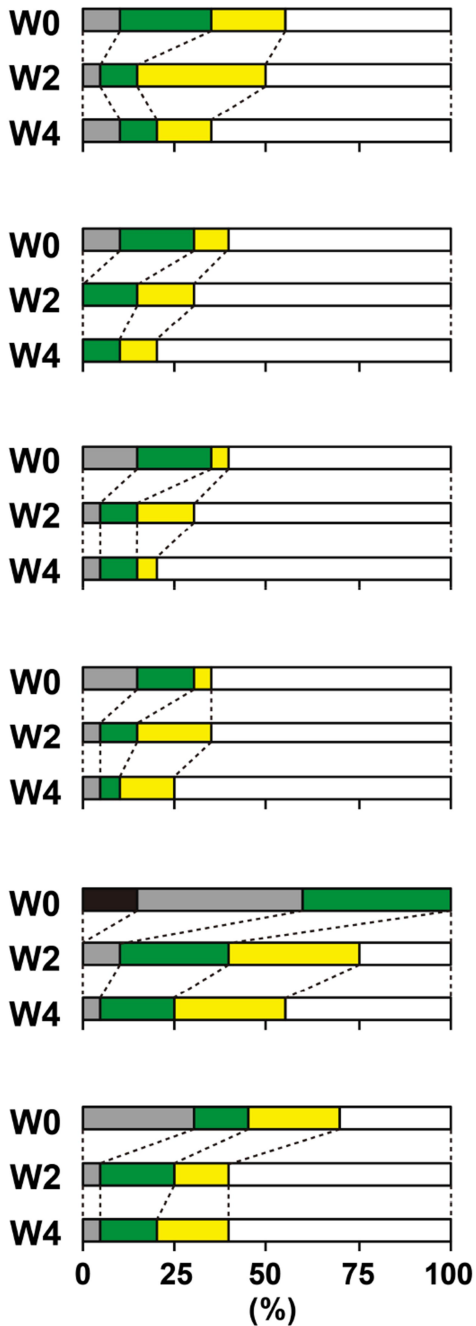

(\%)

\section{Control part}

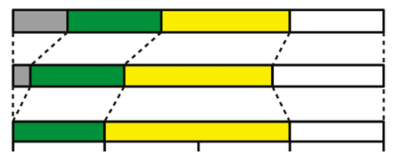

Erythema

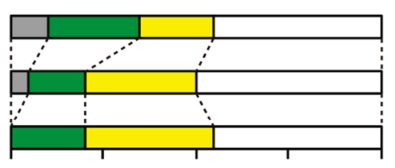

Papules

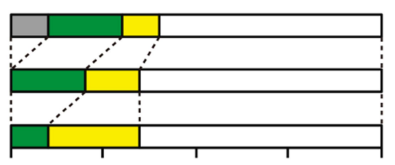

Inflitrations

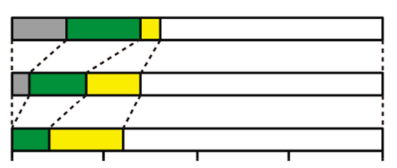

Scars

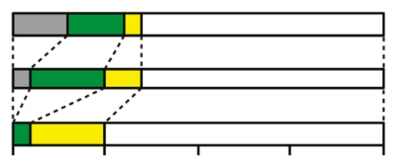

Lichenization

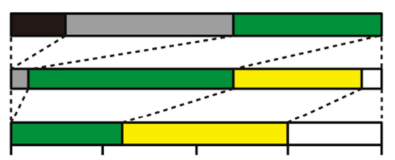

\section{Dryness}

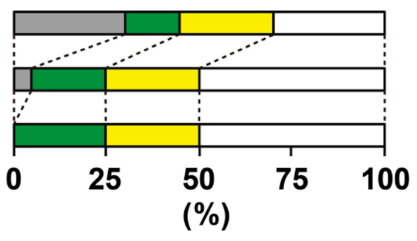

\section{Pruritus}

Severe $\square$ Moderate $\square$ Mild $\square$ Minor $\square$ None

Figure I Changes in the skin condition after the use of MediQOL on the face and body.

Notes: The severity of each skin condition was determined by a dermatologist before and after MediQOL use. A time-dependent change in the skin condition of the face (A) and body (B) was observed. The black, gray, green, yellow, and white bars represent severe, moderate, mild, minor, and none, respectively.

each evaluation item in the test and control parts were not significantly different (Figure 2B).

Most subjects reported relief from worsened skin conditions when tired or stressed (Figure 2). Most subjects also reported relief from burning, tingling, and/or redness in both the face and the body after the use of MediQOL on the test part, while the ratio of the change in the control part was not significant (Figure 2).

\section{Morphology Changes of the Skin Surface} Dryness and/or redness of the skin were observed in the bodies of all subjects at $\mathrm{W} 0$ and were relieved at $\mathrm{W} 4$ in the test part (Figure 3A). A white stratum corneum was observed in nine subjects at W0. It disappeared from the test parts of seven subjects and lessened in the test parts of two subjects at W4, while some white stratum corneum remained on the control part of six subjects at W4 (Figure 

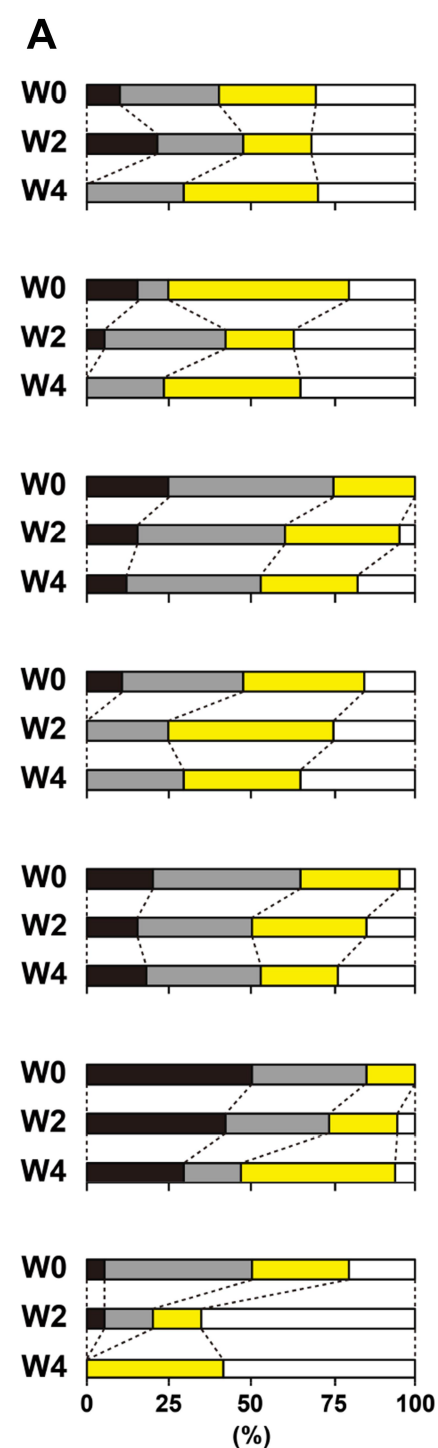
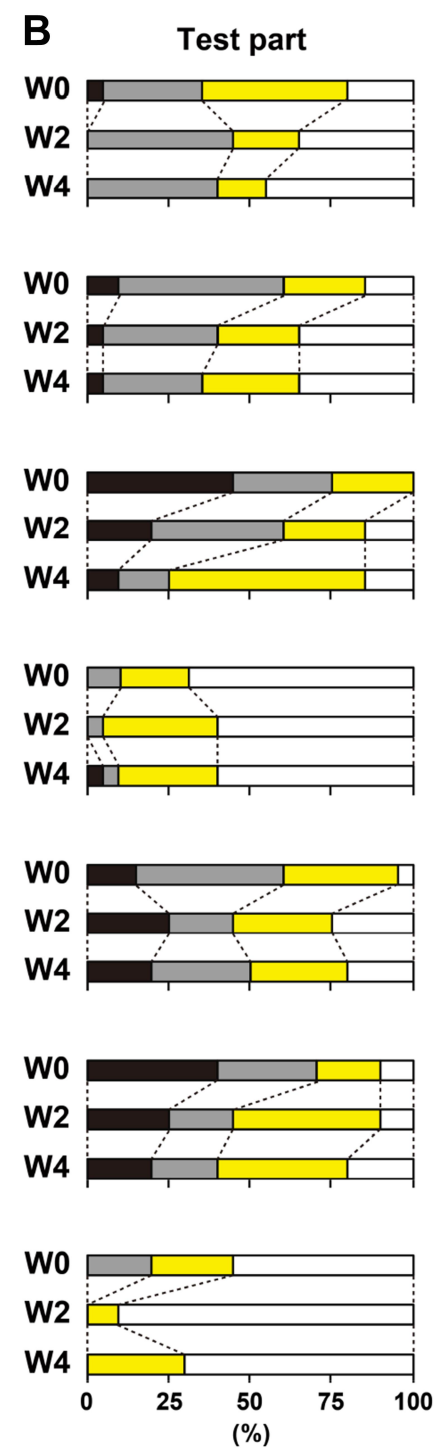

Control part

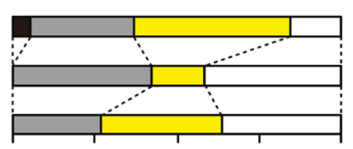

Redness

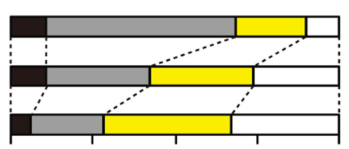

Itchiness

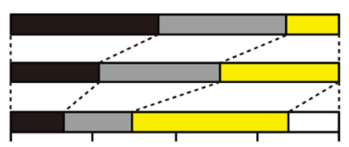

Dryness

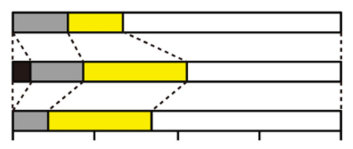

\section{Excess sebum}

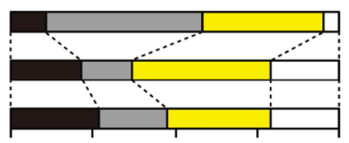

\section{Redness with physical stimulation}

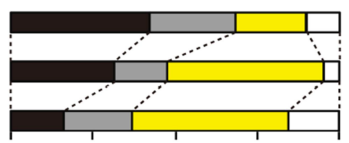

\section{Worsening of skin condition}

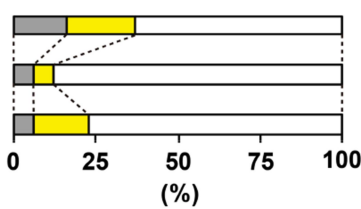

Burning, tingling, and/or redness after the use of MediQOL/cosmetics

\section{All of the time $\square$ Some of the time $\square$ Not very often $\square$ Never}

Figure 2 Subjects' impression of the skin condition.

Notes: Subjective symptoms of each skin condition before and after MediQOL use were checked using a self-description type questionnaire including seven queries with four levels. A time-dependent change in the symptom's severity in the face (A) and body (B) was observed. The black, gray, yellow, and white bars represent all the time, some of the time, not very often, and never, respectively.

3B). Redness of the face was also reduced at W4 (Figure 3C).

\section{Evaluation by Instrumental Analysis}

The average TEWL of the face at W0 was $22.56 \mathrm{~g} / \mathrm{h} / \mathrm{m}^{2}$. Thirty-one and 49 measured values were classified into the moderate and mild groups, respectively, at W0 and W2. However, 25 and 43 measured values were classified into the moderate and mild groups, respectively, at W4, because the facial TEWL data (erythema, skin moisture, and oil content as described below) of three subjects at W4 were excluded. The TEWL of the moderate group decreased during the test period and the TEWL of the mild group increased during the test period; however, neither change was significant (Figure 4A).

The average erythema of the face at W0 was 294.0 arbitrary units (AU). The moderate and mild groups consisted of 39 and 41 measured values at W0 and W2, and 35 and 33 measured values at W4, respectively. Erythema decreased in the moderate group throughout the test period and showed almost no change in the mild group, though transient reduction of erythema was observed at W2 in the mild group (Figure 4B). 
A
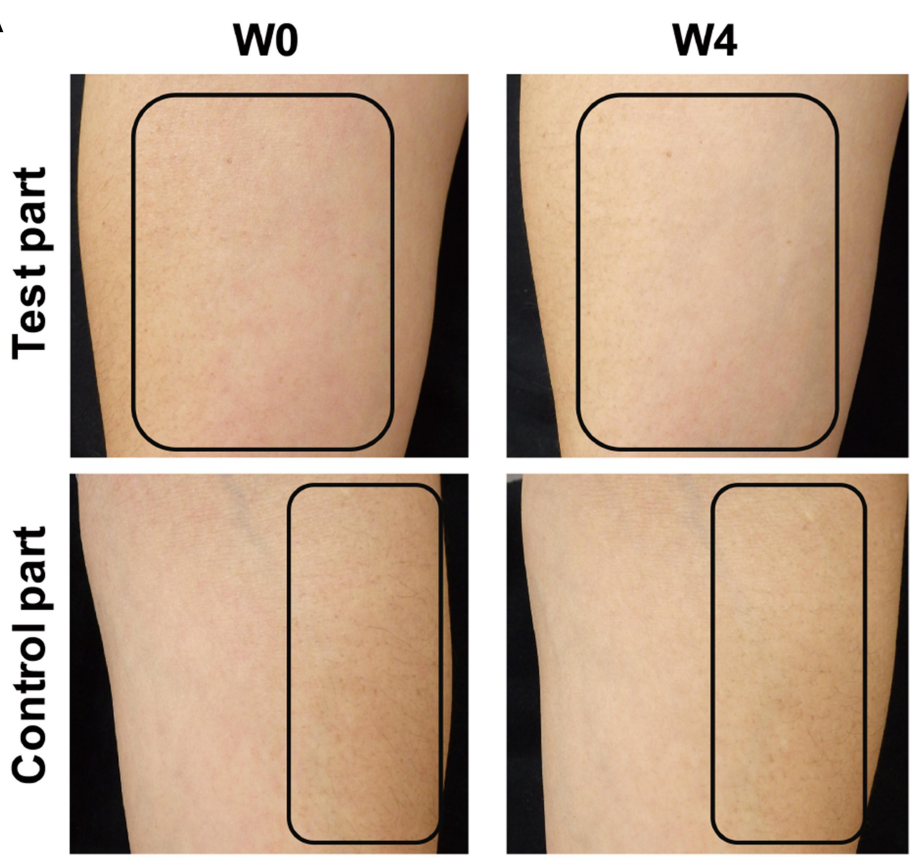

B

wo

W4
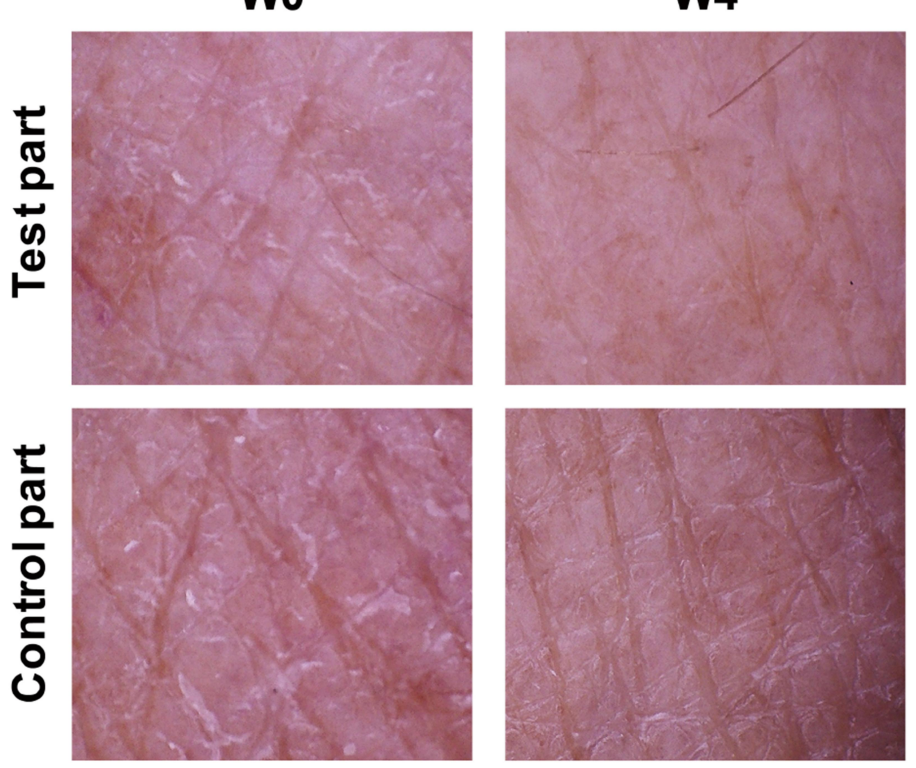

C

wo

W4
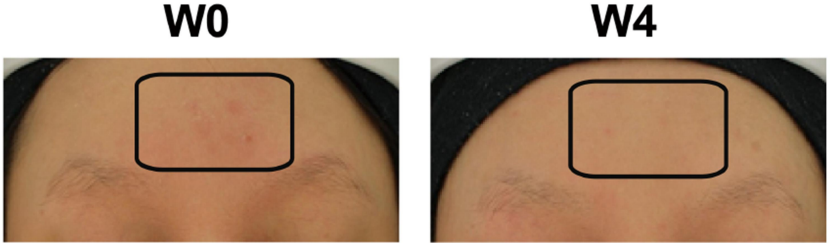

Figure 3 Representative images of the skin before and after MediQOL use.

Notes: Digital camera (A) and microscopic (B) images of a representative subject's arms, and digital images of the robo skin (C) before and after the use of MediQOL are shown. The solid squares in the image data of $A$ and $C$ represent the region with redness and/or dryness. 

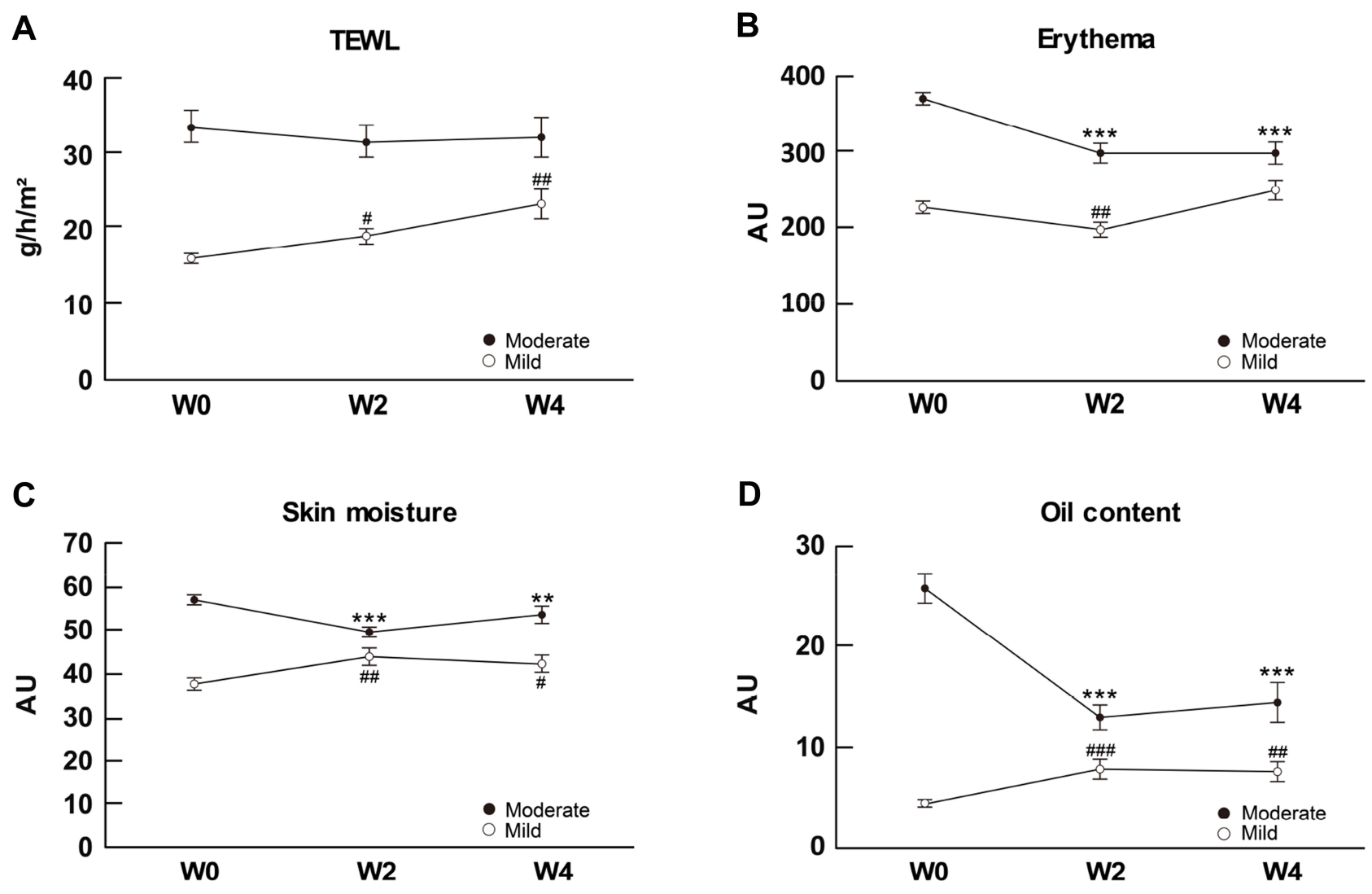

Figure 4 Time-dependent changes in the skin condition of the face after MediQOL use.

Notes: TEWL (A), erythema (B), skin moisture (C), and oil content (D) of the face were measured at W0, W2, and W4. Data are expressed as mean \pm SE. The black and white circles represent the moderate and mild groups, respectively. ${ }^{* *} \mathrm{p}<0.0 \mathrm{I},{ }^{* * *} \mathrm{p}<0.00 \mathrm{I}$ (vs W0 of the moderate group) and ${ }^{\#} \mathrm{p}<0.05,{ }^{\ldots \#} \mathrm{p}<0.0 \mathrm{I},{ }^{\#} \mathrm{p}<0.00 \mathrm{I}$ (vs W0 of the mild group). Abbreviations: AU, arbitrary unit; SE, standard error; TEWL, transepidermal water loss.

The average skin moisture of the face at W0 was 49.8 AU. The moderate and mild groups included 44 and 36 measured values at $\mathrm{W} 0$ and $\mathrm{W} 2$, and 35 and 33 measured values at $\mathrm{W} 4$, respectively. The skin moisture decreased in the moderate group and increased in the mild group throughout the study period (Figure 4C).

The average oil content of the face at W0 was 12.7 AU. Thirty-one and 49 measured values at W0 and W2, and 27 and 41 measured values at W4 were classified into the moderate and mild groups, respectively. The oil content obviously decreased in the moderate group and increased in the mild group throughout the study period (Figure 4D).

The average TEWL of the test part of the body at W0 was $11.25 \mathrm{~g} / \mathrm{h} / \mathrm{m}^{2}$. The moderate and mild groups consisted of 26 and 54 measured values, respectively. The average TEWL of the control part of the body at W0 was $11.07 \mathrm{~g} / \mathrm{h} / \mathrm{m}^{2}$. The moderate and mild groups consisted of 26 and 54 measured values, respectively. In both mild groups, the TEWL of the body hardly changed, while the TEWL of both moderate groups decreased throughout the study period (Figure 5A).

The average erythema of the test part of the body at W0 was 232.7 AU. The moderate and mild groups both consisted of 40 measured values. The average erythema of the control part of the body at W0 was 237.1 AU. The moderate and mild groups consisted of 41 and 39 measured values, respectively. In both mild groups, the erythema of the body did not change, while the erythema of both moderate groups decreased throughout the study period (Figure 5B).

The average skin moisture of the test part of the body at W0 was $46.1 \mathrm{AU}$. The moderate and mild groups consisted of 44 and 36 measured values, respectively. The average skin moisture of the control part of the body at W0 was 44.7 AU. The moderate and mild groups consisted of 43 and 37 measured values, respectively. In both mild groups, the skin moisture of the body increased. The skin moisture of the test part of the body in the moderate groups decreased throughout the study period, while the 


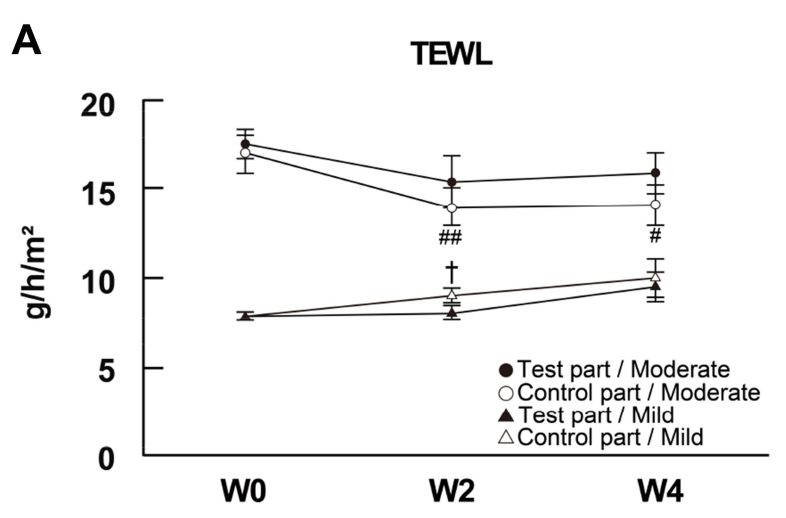

C

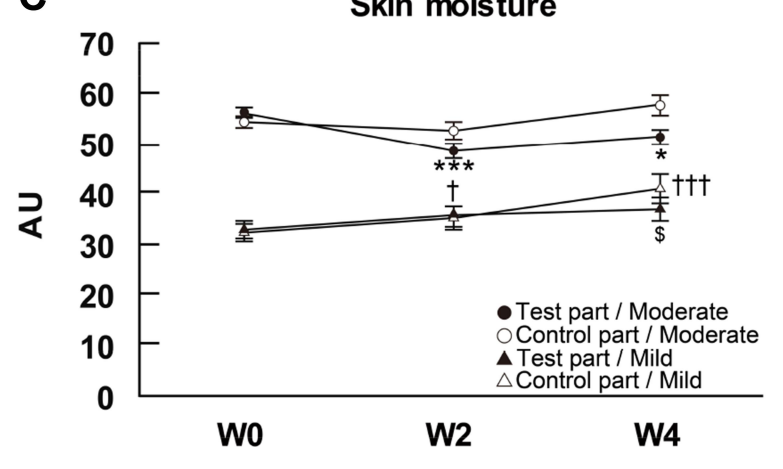

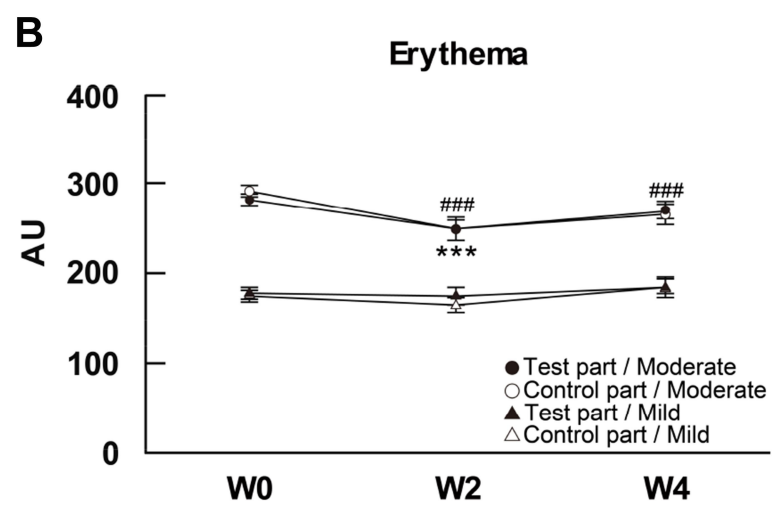

D

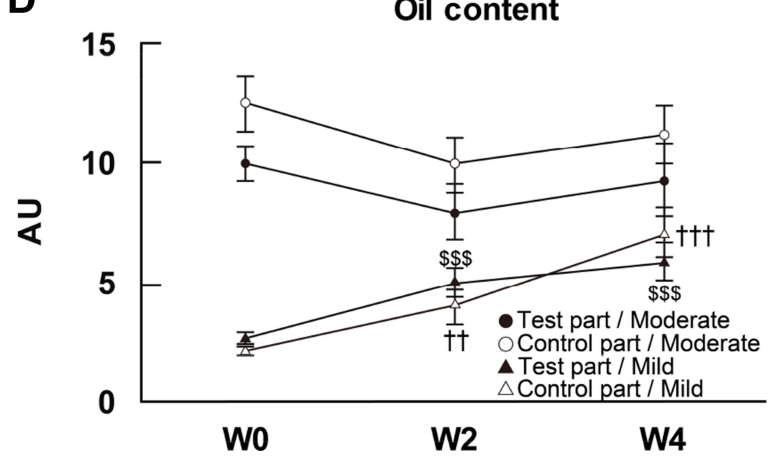

Figure 5 Time-dependent changes in the skin condition of the body after MediQOL use.

Notes: TEWL (A), erythema (B), skin moisture (C), and oil content (D) in the test and control parts of the body were measured at W0, W2, and W4. Data are expressed as mean \pm SE. The black and white circles represent the test and control parts in the moderate group, respectively. The black and white triangles represent the test and control parts in the mild group, respectively. ${ }^{*} p<0.05$, ${ }^{* * *} \mathrm{p}<0.001$ (vs W0 of the moderate group in test part), ${ }^{\#} \mathrm{p}<0.05$, ${ }^{\#} \mathrm{p}<0.01$, ${ }^{\# \#} \mathrm{p}<0.00 \mathrm{I}$ (vs W0 of the moderate group in control part), ${ }^{\$}<<0.05,{ }^{\$ \$} \mathrm{p}<0.001$ (vs W0 of the mild group in test part) and ${ }^{\dagger} p<0.05,{ }^{\dagger \dagger} p<0.01,{ }^{t+\dagger} p<0.001$ (vs W0 of the mild group in control part).

Abbreviations: $\mathrm{AU}$, arbitrary unit; SE, standard error; TEWL, transepidermal water loss.

skin moisture of the control part almost never changed

(Figure 5C).

The average oil content of the test part of the body at W0 was 5.1 AU. The moderate and mild groups consisted of 31 and 49 measured values, respectively. The average oil content of the control part of the body at W0 was 5.4 AU. The moderate and mild groups consisted of 28 and 52 measured values, respectively. In both mild groups, the oil content of the body significantly increased, while the oil content of both moderate groups tended to decrease throughout the study period (Figure 5D).

\section{Safety}

In this study, MediQOL-related adverse events were observed in the face of nine subjects between W2 and W4. The adverse events included skin roughness and redness $(\mathrm{n}=1)$, acne $(\mathrm{n}=1)$, folliculitis $(\mathrm{n}=2)$, redness, itchiness and acne $(n=1)$, tenseness after washing powder use $(n=1)$, itchiness after skin water use $(\mathrm{n}=2)$, and irritation and redness after skin water use $(n=1)$. None of the adverse events were serious and all subjects recovered by the end of the study period or within several days after the end of the study period.

Additionally, one subject developed herpes simplex on the right cheek and another subject developed rough skin on the hand. As the cause of these adverse events was unclear, they were judged as "possibly related".

\section{Discussion}

It is thought that uncomfortable conditions of the skin such as sensitive and dry skin are due to hypofunction of the skin barrier. ${ }^{1-12}$ People with deterioration of the skin barrier function tend to avoid using products that contain unnecessary surfactant, alcohol, preservatives, perfume, coloring matter, and mineral oil, as it seems that these ingredients can lead to further skin irritation, and they prefer cosmetics that are extremely low stimuli and contain the proper ingredients to moisturize the skin. Therefore, we believe that the proper supplementation of the skin components that are lacking at a given time 
should be achieved in subjects with deterioration of the skin barrier function, so we developed a novel cosmetic to replenish the skin barrier.

MediQOL, which was developed to protect against dryness of the skin caused by the reduced skin barrier function and to maintain a good skin condition, is the first skin care cosmetic containing only components that imitate the composition ratio of intercellular lipid and sebum from healthy skin and cell culture mediums. MediQOL does not include any surfactant, alcohol, preservatives, perfume, coloring matter, or mineral oil. In this study, we evaluated the skin condition of subjects with dry skin that requires moisturization before and after MediQOL use.

We found that observed erythema, papules, infiltrations, scars, lichenization, dryness, and pruritus were improved in both the face and the body, and that this improvement was greater in areas that were treated with MediQOL compared to areas treated with other cosmetics. These results indicate that the humidity retention in both the face and the body improves after the use of MediQOL, and that the moisturizing capacity of MediQOL is equal to or greater than that of other cosmetics routinely used by subjects with dry skin.

These observed findings are consistent with the results of the self-reported questionnaire, which revealed that subjects reported a reduction in redness, itchiness, and dryness in both the face and the body after the use of MediQOL. The questionnaire also revealed that subjects felt a decreased worsening of the skin due to tiredness or stress, suggesting that MediQOL can improve the stability of the skin and enhance the subjects' QOL.

We also found that MediQOL resulted in decreased erythema and improved water/oil content, as measured quantitatively. These improvements may be due to the suppression of acute inflammation by the anti-inflammatory agents included in MediQOL (dipotassium glycyrrhizinate and stearyl glycyrrhetinate in MediQOL skin water and skin lipid, respectively). Further, it is predicted that MediQOL washing powder, which contains ingredients similar to sebum, forms a sebum barrier to clean and smooth the skin; MediQOL skin water, which promotes keratinocytes proliferation in vitro (unpublished data), replenishes the skin with nutrition and natural moisturizing ingredients to induce the skin regeneration; and MediQOL skin lipid, which includes fatty acids, ceramides, and cholesterol, recruits lost intercellular lipids to restore barrier function and moisturize the skin. Therefore, continued use of MediQOL allows for the adjustment of the moisture and oil content of the skin, which improves the skin barrier function and in turn suppresses chronic inflammation and reduces responses to external stimuli. The quantitative data obtained in this study also correlate to the skin observation and selfreported questionnaire data.

The TEWL of the face and body was decreased in the moderate groups, while the TEWL of the body was not changed in the mild group. However, the TEWL of the face increased in the mild group. This increase may be due to the transient adverse events observed in subjects using MediQOL (described below). These adverse events affect the recovery of the skin barrier function, as increased TEWL of the face was found in five of nine subjects who experienced adverse events. Also, the turnover cycle of the horny layer lasts 28-45 days, depending on the subject's age. Therefore, the recovery of the skin barrier function may not have been completed during the study period.

Nine subjects experienced adverse events in the face that were related to MediQOL between W2 and W4. As the adverse events were transient and not serious, they may have been due to the subject's health, the climate, or the skin condition on that specific day. In brief, the skin conditions induced by MediQOL were generally well tolerated by subjects with dry skin. However, we cannot exclude the possibility that MediQOL has a direct adverse effect on the skin. These adverse events may be prevented by adjusting the frequency or combination of MediQOL use at the first sign of adverse symptoms.

Our study results, which demonstrated the effect of humidity retention in subjects with dry skin using cosmetics consisting only of imitated components of the healthy skin composition, are promising and we believe that MediQOL meets a demand for people with impaired skin barrier function, although MediQOL leaves room for some improvement for reducing irritation to the skin. In addition, we expect that MediQOL will also be useful for the skin care in people with skin diseases. At present, there are many people who are troubled by skin conditions and seek ideal cosmetics which have low irritancy to the skin. Therefore, we hope to continue to develop and provide cosmetics that hardly cause skin irritancy and ameliorate skin conditions.

\section{Conclusion}

MediQOL improves the skin condition in subjects with dry skin by regulating the moisture retention and balance of the 
water and oil content of the skin. This novel cosmetic enhances QOL for subjects with dry skin and is expected to be effective for target persons with various skin types, including oily, mixed, and sensitive skin as well as dry skin.

\section{Data Sharing Statement}

The authors do not intend to share individual de-identified participant data separately.

\section{Acknowledgments}

We thank Yoshiko Suzuki and Toshiko Matsuo of Nomura Dermatology Clinic for their cooperation in this study.

\section{Funding}

This study was supported financially by NANOEGG ${ }^{\circledR}$ Research Laboratories, Inc., Kanagawa, Japan.

\section{Disclosure}

Kazunori Tanaka, Teruaki Nagasawa, Yoshiki Kubota, Akihiko Miyake and Kimio Kawamura are employees of NANOEGG® Research Laboratories, Inc., Kanagawa, Japan. Yoko Yamaguchi owns stocks or shares in NANOEGG® Research Laboratories, Inc., Kanagawa, Japan. Yuko Nomura is a dermatologist and a director of the Nomura Dermatology Clinic, Kanagawa, Japan. The authors report no other conflicts of interest in this work.

\section{References}

1. Misery L, Ständer S, Szepietowski JC, et al. Definition of sensitive skin: an expert position paper from the special interest group on sensitive skin of the international forum for the study of itch. Acta Derm Venereol. 2017;97(1):4-6. doi:10.2340/00015555-2397
2. Misery L, Loser K, Ständer S. Sensitive skin. $J$ Eur Acad Dermatol Venerol. 2016;30(Suppl. 1):2-8. doi:10.1111/jdv.13532

3. Hashimoto K, Nokami N, Matsushita N. Sensitive skin and questionnaire in patients. Fragr J. 2016;44(9):72-75.

4. Misery L, Weisshaar E, Brenaut E, et al. Pathophysiology and management of sensitive skin: position paper from the special interest group on sensitive skin of the International Forum for the Study of Itch (IFSI). J Eur Acad Dermatol Venereol. 2020;34(2):222-229. doi: $10.1111 / j \mathrm{dv} .16000$

5. Wan DC, Wong VW, Longaker MT, Yang GP, Wei FC. Moisturizing different racial skin types. $J$ Clin Aesthet Dermatol. 2014;7(6):25-32.

6. Purnamawati S, Indrastuti N, Danarti R, Saefudin T. The role of moisturizers in addressing various kinds of dermatitis: a review. Clin Med Res. 2017;15(3-4):75-87. doi:10.3121/cmr.2017.1363

7. Dawnielle C, Endly DO, Miller RA. Oily skin: a review of treatment options. J Clin Aesthet Dermatol. 2017;10(8):49-55.

8. Seidenari S, Francomano M, Mantovani L. Baseline biophysical parameters in subjects with sensitive skin. Contact Dermat. 1998;38 (6):311-315. doi:10.1111/j.1600-0536.1998.tb05764.x

9. Pinto P, Rosado C, Parreirao C, Rodrigues LM. Is there any barrier impairment in sensitive skin?: a quantitative analysis of sensitive skin by mathematical modeling of transepidermal water loss desorption curves. Skin Res Technol. 2011;17(2):181-185. doi:10.1111/j.16000846.2010.00478.x

10. Farage MA. The prevalence of sensitive skin. Front Med. 2019;6:98. doi: $10.3389 /$ fmed.2019.00098

11. Lodén M. The clinical benefit of moisturizers. J Eur Acad Dermatol Venereol. 2005;19(6):672-688. doi:10.1111/j.1468-3083.2005.01326.x

12. Lynde CW. Moisturizers: what they are and how they work. Skin Therapy Lett. 2001;6(13):3-5.

13. Elias PM, Choi EH. Interactions among stratum corneum defensive functions. Exp Dermatol. 2005;14(10):719-726. doi:10.1111/j.16000625.2005.00363.x

14. Feingold KR. The role of epidermal lipids in cutaneous permeability barrier homeostasis. J Lipid Res. 2007;48(12):2531-2546. doi:10.1194/j1r.R700013-JLR200

15. Feinglod KR. Lamellar bodies: the key to cutaneous barrier function. $J$ Invest Dermatol. 2012;132(8):1951-1953. doi:10.1038/ jid.2012.177

16. Werz PW. Lipids and the permeability and antimicrobial barriers of the skin. J Lipids. 2018;5954034.
Clinical, Cosmetic and Investigational Dermatology is an international, peer-reviewed, open access, online journal that focuses on the latest clinical and experimental research in all aspects of skin disease and cosmetic interventions. This journal is indexed on CAS.
The manuscript management system is completely online and includes a very quick and fair peer-review system, which is all easy to use. Visit http://www.dovepress.com/testimonials.php to read real quotes from published authors. 